\title{
Topology Control and Opportunistic Routing for Underwater Acoustic Sensor Networks
}

\author{
Rodolfo W. L. Coutinho ${ }^{1,2}$, Azzedine Boukerche ${ }^{2}$, Luiz Vieira ${ }^{1}$ and Antonio A. F. Loureiro ${ }^{1}$ \\ ${ }^{1}$ Depto. de Ciência da Computação - Universidade Federal de Minas Gerais (UFMG) \\ Av. Antônio Carlos, 6627 - Belo Horizonte, MG - Brazil \\ ${ }^{2}$ School of Electrical Engineering and Computer Science - University of Ottawa \\ 800 King Edward Ave. - Ottawa, ON - Canada \\ \{rwlc, loureiro, lfvieira\}@dcc.ufmg.br, boukerch@eecs.uottawa.ca
}

\begin{abstract}
Underwater wireless sensor networks (UWSNs) are emerging to enable large-scale ocean monitoring with the goal of reducing the human knowledge gap of underwater environments and the life underneath them. However, several challenges still limit the deployments of UWSNs to small-scale and confined underwater monitoring applications. The goals of this thesis is to investigate and develop analytical models, algorithms and protocols in order to tackle the fundamental data communication challenge in the underwater environment, and advance the state-of-the-art towards feasible large-scale deployment of UWSN applications.
\end{abstract}

\section{Introduction}

Oceans cover two-thirds of the Earth's surface and are vital for supporting life on our planet. However, currently we have explored no more than 5\% of their volume [National Ocean Service 2018]. Indeed, we do understand more about entities and phenomena outside our planet than we do about our oceans and the life underneath them. Worryingly, current technologies for large-scale aquatic monitoring are not at a mature stage enough to change this despairing reality.

Significant research efforts have been conducted towards the development of underwater sensor networks (UWSNs) [Heidemann et al. 2012] (Fig. 1a) for large-scale ocean monitoring. The Defense Advanced Research Projects Agency (DARPA), for instance, announced in 2017 the "Ocean of Things program" (Fig. 1b), which aims to develop small low-cost floats that will form large-scale distributed underwater sensor networks to enable persistent maritime situational awareness. However, despite the current and expected advances in underwater sensing technologies, the efficient underwater wireless data communication is still a fundamental and critical problem that needs to be further investigated and experimented. This is because of the challenges imposed by the underwater acoustic communication, which includes limited bandwidth, low reliable data delivery, high delay and energy cost, as well as the challenges imposed by the underwater environment, such as high noise and mobility.

\subsection{Objective and Contributions}

This work was then motivated and inspired by the need for reliable and efficient underwater wireless data delivery in UWSNs, which is a research challenge that makes high-costly

\footnotetext{
${ }^{1}$ https://www.darpa.mil/news-events/2017-12-06
} 


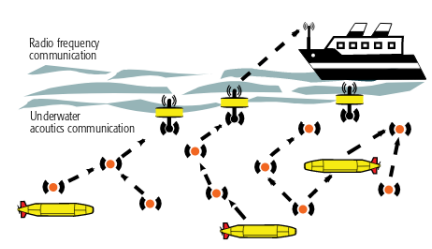

(a) UWSN architecture

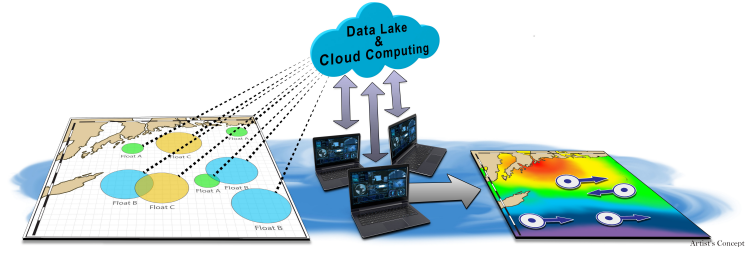

(b) Ocean of Things

Figure 1. Underwater sensor networks.

and unfeasible the deployment of large-scale UWSN applications. To tackle this problem, the research conducted in the scope of this thesis was broadly divided into two parts. The first one investigates how to increase the data delivery reliability in UWSNs whereas the second part studies how to reduce the energy cost of underwater wireless data communication in UWSNs. These two parts complement each other, as we will see below. In summary, we improved the data delivery reliability and reduced the energy communication cost in UWSNs by developing stochastic-based analytical models, centralized and distributed topology control algorithms and geographic and opportunistic routing protocols. This research advanced the start-of-the-art by (i) proposing and modeling an innovative mobility-assisted methodology for the design of communication void handling algorithms for geographic and opportunistic routing in UWSNs, (ii) developing a suite of geographic and opportunistic routing protocols aimed to increase data delivery and reduce energy consumption in UWSNs, (iii) developing guidelines for further design of opportunistic routing protocols for UWSNs, as well as a framework to classify topology control works in UWSNs, $(i v)$ proposing and modeling opportunistic routing and duty-cycling in UWSNs, as well as the on-the-fly adjustment of the sleep interval of underwater sensor nodes to balance the energy consumption among them, and $(v)$ developing a mathematical framework to study power control and opportunistic routing in UWSNs.

\section{Background}

Before we proceed further discussing the contributions of this thesis in terms of mathematical models, algorithms and protocols, it is important to highlight the thesis' contributions in terms of guidelines that it provided for future research. This is an equally important contribution in the sense that it will save time and energy of future researchers and practitioners that will indeed to design and implement routing protocols and topology control algorithms for UWSN applications. First, this thesis originated an in-depth survey of topology control in UWSNs (refer to contributions list, item 1). The survey developed a general framework for the design of topology control algorithms in UWSNs (Fig. 2a) and then classified the current state-of-the-art topology control protocols into power control-, wireless interface management- and mobility assisted-based topology control (Fig. 2b).

Second, this thesis proposed guidelines for further design opportunistic routing (OR) protocols in UWSNs (refer to contributions list, item 2). It identified the two main building blocks that must be implemented when proposing OR protocols for UWSNs. After that, it provided distinct techniques that could be considered to implement each building block. Finally, it drew a qualitative and quantitative critical discussion of the highlighted distinct techniques from results obtained from the simulation of different UWSN application scenarios. 


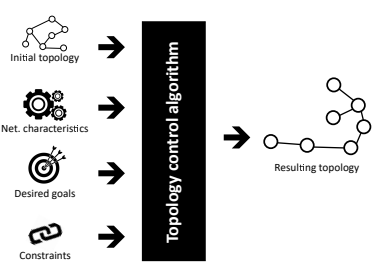

(a) Proposed framework

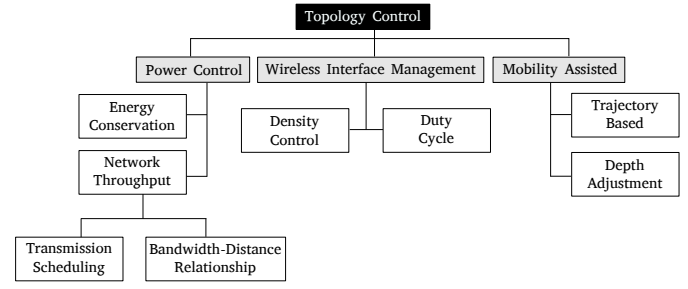

(b) Proposed classification

Figure 2. Topology control for underwater sensor networks.

In addition, this thesis debated the major networking tasks that significantly consume energy in UWSNs, such as data aggregation, routing path discovery, medium access and data transmission over long distances. Indeed, it highlighted state-of-the-art approaches proposed to conserve energy at different networking layers of UWSNs (refer to contributions list, item 3).

Finally, the achievements and knowledge produced by this research were presented in two tutorials in international conferences (IEEE DCOSS 2017 and ACM MSWiM 2017). These tutorials were delivered to an audience composed of undergrad and graduate students, as well as professors that had diverse backgrounds in terms of networking, modeling and protocol design (refer to contributions list, items 4 and 5). The aforementioned contributions of this thesis were reported in the following publications:

1. R. W. L. Coutinho, A. Boukerche, L. F. M. Vieira, A. A. F. Loureiro, "Underwater Wireless Sensor Networks: A New Challenge for Topology Control-Based Systems”, ACM Computing Surveys, 51(1):19:1-19:36, 2018. [Qualis A1, Impact factor: 6.748]

2. R. W. L. Coutinho, A. Boukerche, L. F. M. Vieira, A. A. F. Loureiro, "Design guidelines for opportunistic routing in underwater networks", IEEE Communications Magazine, 54(2):40-48, 2016. [Qualis A1, Impact factor: 10.435]

3. R. W. L. Coutinho, A. Boukerche, L. F. M. Vieira, A. A. F. Loureiro, "On the design of green protocols for underwater sensor networks". IEEE Communications Magazine, 54(10):67-73, 2016. [Qualis A1, Impact factor: 10.435]

4. R. W. L. Coutinho, A. Boukerche and A. A. F. Loureiro, "Underwater Wireless Sensor Networks For Ocean Monitoring Missions", The Philosophy of Mission-Oriented Wireless Sensor Networks, Springer, 2018. To appear. [Book chapter]

5. R. W. L. Coutinho, A. Boukerche. "Opportunistic routing in underwater sensor networks: Potentials, challenges and guidelines", In Proc. of the 13th Int'l Conference on Distributed Computing in Sensor Systems (DCOSS), 1-2, 2017. [Qualis A2, Conference]

6. R. W. L. Coutinho, A. Boukerche. "Data collection in underwater wireless sensor networks: Research challenges and potential approaches", In Proc. of the 20th ACM Int'l Conf. on Modeling, Analysis and Simulation of Wireless and Mobile Systems (MSWiM), 5-8, 2017. [Qualis B1, Conference]

\section{Reliable Data Collection in UWSNs}

The first part of the thesis developed solutions to increase the data delivery rate in the loosely underwater communication environment of UWSNs. As will be discussed in the following, this goal was achieved by the design of an innovative methodology for communication void handling ${ }^{2}$ and a suite of novel location-based geographic and opportunistic routing protocols for non-mobile and mobile multi-sink UWSNs.

\subsection{Mobility-Assisted Void Handling}

The state-of-the-art protocols regarding void handling algorithms for UWSNs, by the time of this thesis, were based on the principle of bypassing the communication void area or increasing the communication range. When the bypassing void region-based principle is

\footnotetext{
${ }^{2}$ Communication void handling algorithms are designed to route data packets from void nodes or communication void areas that occur in geographic routing protocols.
} 
considered, a void node discovers and maintains an alternative path to a node that can resume the greedy geographic routing. When the power control-based principle is considered, a void node increases its transmission power in order to reach novel neighboring nodes that can resume the greedy forward routing. Both principles increase the energy consumption of UWSNs, because of the high-energy cost needed to discover and maintain a new routing path, as well as to transmit among long-distant loosely underwater acoustic links.

Therefore, this thesis proposed, modeled and evaluated an innovative mobilityassisted principle that relies on the controlled depth adjustment of void nodes. When the proposed mobility-assisted principle is considered, a void node controllably moves to a new depth where it can resume the greedy geographic and opportunistic data packet forwarding towards surface sink nodes. This developed principle to tackle the communication void region problem in UWSNs was a innovative solution that opened a new research direction. It received the best paper award at the IEEE MASCOTS conference, a top-tier venue for modeling, analysis, and simulations. Moreover, it has been inspiring recent designs of void handling protocols for non-mobile and mobile underwater sensor networks. These contributions were reported in the following publications:

7. R. W. L. Coutinho, A. Boukerche, L. F. M. Vieira, A. A. F. Loureiro, "A novel void node recovery paradigm for long-term underwater sensor networks", Ad Hoc Networks, 34:144-156, 2015. [Qualis A2, Impact factor: 3.047]

8. R. W. L. Coutinho, A. Boukerche, L. F. M. Vieira, A. A. F. Loureiro, "Performance modeling and analysis of void-handling methodologies in underwater wireless sensor networks", Computer Networks, 126:1-14, 2017. [Qualis A1, Impact factor: 2.516]

9. R. W. L. Coutinho, L. F. M. Vieira, A. A. F. Loureiro, "Movement Assisted-topology Control and Geographic Routing Protocol for Underwater Sensor Networks", In Proc. of the IEEE 22nd Int'1 Symposium on Modelling, Analysis Simulation of Computer and Telecommunication Systems (MASCOTS), 112-119, 2014. Best Paper Award. [Qualis A2, Conference]

10. R. W. L. Coutinho, L. F. M. Vieira, A. A. F. Loureiro, "Movement Assisted-topology Control and Geographic Routing Protocol for Underwater Sensor Networks", In Proc. of the 16th ACM Int'l Conference on Modeling, Analysis \& Simulation of Wireless and Mobile Systems (MSWiM), 189-196, 2013. [Qualis B1, Conference]

\subsection{Geographic and Opportunistic Routing}

To overcome the limitations and costs of traditional proactive and reactive routing in UWSNs, this thesis proposed, modeled and developed a suite of geographic and opportunistic routing (GOR) protocols for mobile and non-mobile multi-sink UWSNs. To the best of our knowledge, the designed protocols were the first in the literature to consider the position location, instead of the depth information, of underwater sensor nodes for directed geographic and opportunistic routing towards multi-sinks at the ocean surface. The use of directional routing for multi-sinks and the opportunistic packet forwarding at each hop led the proposed protocols to improve the underwater acoustic communication reliability and outperform related proposals. The proposed protocols were reported in the following contributions:

11. R. W. L. Coutinho, A. Boukerche, L. F. M. Vieira, A. A. F. Loureiro, "Geographic and Opportunistic Routing for Underwater Sensor Networks", IEEE Transactions on Computers, 65:548-561, 2016. [Qualis A1, Impact factor: 2.916]

12. R. W. L. Coutinho, A. Boukerche, L. F. M. Vieira, A. A. F. Loureiro, "REnOR:Energy Balanced Routing Protocol for Underwater Senor Networks", IEEE Transactions on Sustainable Computing, Under review.

13. R. W. L. Coutinho, A. Boukerche, L. F. M. Vieira, A. A. F. Loureiro, "EnOR: Energy balancing routing protocol for underwater sensor networks", In Proc. of the IEEE Int'1 Conference on Communications (ICC), 1-6, 2017. [Qualis A1, Conference]

14. R. W. L. Coutinho, A. Boukerche, L. F. M. Vieira, A. A. F. Loureiro, "GEDAR: Geographic and opportunistic routing protocol with Depth Adjustment for mobile underwater sensor networks", In Proc. of the IEEE Int'l Conference on Communications (ICC), 215-256, 2014. [Qualis A1, Conference]

15. R. W. L. Coutinho, A. Boukerche, L. F. M. Vieira, A. A. F. Loureiro, "Um protocolo de roteamento para o consumo balanceado de energia em redes de sensores aquáticas", In Proc. of the Simpósio Brasileiro de Redes de Computadores e Sistemas Distribuídos (SBRC), 1-14, 2017. [Qualis B2, Conference]

16. R. W. L. Coutinho, L. F. M. Vieira, A. A. F. Loureiro, "DCR: Depth-Controlled Routing protocol for underwater sensor networks", In Proc. of the IEEE Int'l Symposium on Computers and Communications (ISCC), 453-458, 2013. [Qualis A2, Conference] 


\section{Energy-Efficient Data Collection in UWSNs}

The second part of this thesis developed solutions to conserve energy in scenarios of energy-hungry UWSNs. It proposed novel duty-cycling and power control protocols for the particular scenario of underwater acoustic communication and environment, as the classical and well investigated approaches for duty-cycling and power control cannot be applied in UWSNs, due to the characteristics of the underwater acoustic channel and the challenges for synchronization and transmission scheduling in UWSNs. As will see in the following, we innovatively designed and developed symbiotic duty-cycling and opportunistic routing, as well as power control and opportunistic routing, towards energyefficient UWSN applications.

\subsection{Duty-Cycling}

Duty cycling has been extensively studied as an approach to conserve energy in terrestrial wireless sensor networks (WSNs). However, the classical solutions designed for WSNs will diminish the performance of UWSNs, instead of improving it, because of the limited bandwidth, low quality of acoustic links and the high-energy consumption for transmissions. For instance, the use of traditional asynchronous duty-cycling in UWSNs may worsen the energy consumption, instead of reduce it, because of the high energy cost, of the order of Watts, for packet transmissions in the underwater acoustic channel and the need of periodic transmissions for nodes' duty-cycle schedules exchange.

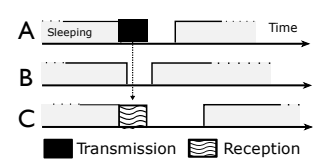

(a) Naive

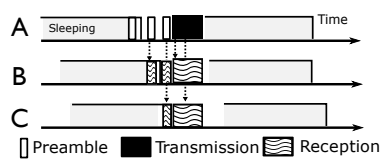

(b) Low Power Listening

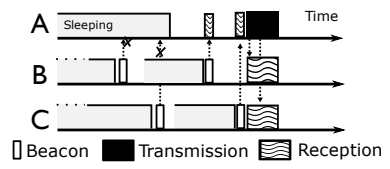

(c) Low Power Probing

Figure 3. Symbiotic design of duty-cycling and opportunistic routing for UWSNs.

This thesis proposed and modeled three novel methodologies (Fig. 3) for asynchronous duty-cycling towards energy-efficient and reliable UWSNs. It innovatively proposed the symbiotic design of duty-cycling and opportunistic routing for UWSNs. Hence, the use of multiple nodes as next-hop candidates reduces the need for duty-cycling schedule exchanges and keeps the effective network density at a proper level that guarantees an acceptable data delivery rate. In addition, this thesis developed an optimization model to balance the energy consumption of the underwater sensor nodes, by adjusting on-the-fly the sleep interval at duty-cycled underwater sensor nodes. The results of this research were presented in the following publications:

17. R. W. L. Coutinho, A. Boukerche, L. F. M. Vieira, A. A. F. Loureiro, "Joint Duty-Cycling and Opportunistic Routing for Sustainable Underwater Sensor Networks", IEEE Transactions on Sustainable Computing, Under review.

18. R. W. L. Coutinho, A. Boukerche, L. F. M. Vieira, A. A. F. Loureiro, "A Novel Centrality Metric for Topology Control in Underwater Sensor Networks", In Proc. of the 19th ACM Int'l Conference on Modeling, Analysis and Simulation of Wireless and Mobile Systems (MSWiM), 205-212, 2016. [Qualis B1, Conference]

19. R. W. L. Coutinho, A. Boukerche, L. F. M. Vieira, A. A. F. Loureiro, "Modeling the sleep interval effects in duty-cycled underwater sensor networks", In Proc. of the IEEE International Conference on Communications (ICC), 1-6, 2016. [Qualis A1, Conference]

20. R. W. L. Coutinho, A. Boukerche, L. F. M. Vieira, A. A. F. Loureiro, "Modeling and Analysis of Opportunistic Routing in Low Duty-Cycle Underwater Sensor Networks", In Proc. of the 18th ACM Int'l Conference on Modeling, Analysis and Simulation of Wireless and Mobile Systems (MSWiM), 125-132, 2015. [Qualis B1, Conference] 


\subsection{Power Control}

Finally, this thesis proposed a novel principle for power control in UWSNs. In the proposed principle, power control is jointly performed with opportunistic routing, that is the decision regarding the transmission power in the nodes considers the possible number of candidates and the quality of them, the node will have with the corresponding communication range. We proposed an analytical framework to study whether the transmission power of a node should be increased or not to include additional neighboring nodes in the opportunistic routing next-hop forwarding candidate set. More specifically, the aggregate quality of the new set of neighboring nodes, which will reflect in the data delivery reliability, and the energy cost are considered to decide what transmission power level an underwater sensor node should use. This contribution is presented in the following publication:

21. R. W. L. Coutinho, A. Boukerche, A. A. F. Loureiro, "Modeling Power Control and Anypath Routing in Underwater Acoustic Sensor Networks", In Proc. of the IEEE Wireless Communications and Networking Conference (WCNC), 125132, 2018. [Qualis B1, Conference]

\section{Research Accomplishments}

The main accomplishments of this research can be summarized as follows:

- Eight papers in top-tier IEEE, ACM and Elsevier periodic journals and one book chapter. In the list of journal papers, six are already published and two are under review.

- Ten papers in IEEE and ACM international conferences, where one of them received the best paper award in the IEEE MASCOTS.

- Two tutorials in international conferences on modeling and performance evaluation of networking and distributed systems.

\section{Conclusion and Thesis Impact}

This thesis tackled the efficient data communication challenge in UWSNs, which is one of the fundamental problems that need to be solved towards the large-scale deployment of UWSN applications. Herein, we proposed, designed and developed mathematical models, topology control algorithms, and geo-opportunistic routing protocols for reliable data delivery and energy-efficient UWSNs. The knowledge produced from this research has been featured in several top-tier venues in the area, either in terms of scientific publications or in delivered tutorials. The results of this thesis have impacted the literature in several ways. Our innovative mobility-assisted topology control has been inspiring innovative designs of topology control algorithms and routing protocols for UWSNs. Our survey paper provided a critical and comprehensive review of the literature while our models provided important insights that have been guiding the development of novel protocols for UWSNs, as corroborated for the almost three hundred citations our contributions received in this brief period.

\section{References}

Heidemann, J., Stojanovic, M., and Zorzi, M. (2012). Underwater sensor networks: applications, advances and challenges. 370(1958):158-175.

National Ocean Service (2018). How much of the ocean have we explored? https: / / oceanservice.noaa.gov/facts/exploration.html. Online; accessed February 2018. 